\title{
FACE NUMBERS OF PSEUDOMANIFOLDS WITH ISOLATED SINGULARITIES
}

\author{
ISABELLA NOVIK* ${ }^{*}$ and ED SWARTZ ${ }^{\dagger}$
}

\begin{abstract}
We investigate the face numbers of simplicial complexes with Buchsbaum vertex links, especially pseudomanifolds with isolated singularities. This includes deriving Dehn-Sommerville relations for pseudomanifolds with isolated singularities and establishing lower and upper bound theorems when the singularities are also homologically isolated. We give formulas for the Hilbert function of a generic Artinian reduction of the face ring when the singularities are homologically isolated and for any pure two-dimensional complex. Some examples of spaces where the $f$-vector can be completely characterized are described. We also show that the Hilbert function of a generic Artinian reduction of the face ring of a simplicial complex $\Delta$ with isolated singularities minus the $h$-vector of $\Delta$ is a PL-topological invariant.
\end{abstract}

\section{Introduction}

Stanley's introduction of commutative algebra into the study of face numbers of simplicial complexes via the face ring revolutionized the subject. For instance, he was able to give a complete characterization of all possible $f$-vectors of Cohen-Macaulay complexes [28]. A simplicial complex is Cohen-Macaulay (CM) if the homology of the link of any face (including the empty face) is trivial except possibly in the top dimension. Thus all triangulated spheres and balls are CM complexes. In [25] Schenzel showed how to extend many of these ideas to Buchsbaum complexes, that is, pure complexes whose vertex links are CM. This includes triangulations of manifolds (with and without boundary). In [18] algebraic aspects of face rings of spaces with more complicated singularities were studied.

The algebraic picture is, roughly, the following: a simplicial complex $\Delta$ is $\mathrm{CM}$ if and only if all local cohomology modules of the face ring of $\Delta$ but the top one vanish; $\Delta$ is Buchsbaum if and only if all local cohomology modules of the face ring (but the top one) vanish except in degree zero; and $\Delta$ has singularity dimension at most $m-1$ if and only if all local cohomology modules (but the

\footnotetext{
* Research partially supported by Alfred P. Sloan Research Fellowship and NSF grant DMS0801152.

${ }^{\dagger}$ Research partially supported by NSF grant DMS-0900912.

Received 17 November 2010, in final form 16 August 2011.
} 
top one) of the face ring of $\Delta$ modulo $m$ generic linear forms vanish except in degrees $0,1, \ldots, m$. What are the dimensions of the non-vanishing parts of these local cohomology modules and what is the module structure? In the Buchsbaum case the answer is well-known [25]: the dimensions coincide with the Betti numbers of $\Delta-$ the dimensions of (reduced) simplicial homology of the complex, and the module structure is trivial. However, for singular spaces the answer is much less tractable at the moment, and that is what makes studying their face numbers a significantly harder problem.

Here we begin work on this problem. Specifically we begin the difficult task of understanding the precise connection between the algebraic, enumerative and combinatorial properties of face rings of singular spaces: we examine face rings of pure complexes whose vertex links are Buchsbaum with a particular emphasis on pseudomanifolds with isolated singularities. Pseudomanifolds with isolated singularities provide a vast generalization of manifolds, and include, among others, many quotients of compact manifolds where the group action is free away from a finite set, such as Kummer varieties, and complexes obtained from compact manifolds with boundary by coning off boundary components, such as suspensions. In particular, face enumeration on manifolds with boundary is closely related to face enumeration on spaces with isolated singularities. For a specific example see [21, Theorem 5.1].

We start by introducing the basic notations, definitions and results we need for simplicial complexes, face rings and local cohomology. In Section 3 we derive Dehn-Sommerville relations for pseudomanifolds with isolated singularities. This is followed by a close examination of spaces with homologically isolated singularities, see Section 4 for a definition. In this case we are able to establish formulas for the Hilbert function of an arbitrary Artinian reduction of the face ring (Theorems 4.2 and 4.7), a version of the upper bound theorem (Theorem 4.10), and a lower bound formula (Theorem 4.9) analogous to [22, Theorem 5.2] for manifolds. The latter result provides a considerable strengthening of Barnette's lower bound theorem [3], [12] by taking into account homological data. We remark that having homologically isolated singularities is a topological invariant of a space; in particular, our results apply to all triangulations of spaces having this property.

Highly connected (in the topological sense) spaces are the subject of Section 5. A corollary of these results is a formula for the Hilbert function of generic Artinian reductions of pure two-dimensional complexes (Eq. (12)). Some examples of pseudomanifolds where we can give a complete characterization of their $f$-vectors are the subject of Section 6. Finally, we show that the Hilbert function of a generic Artinian reduction of the face ring of a complex with isolated singularities is determined by its $f$-vector and PL-homeomorphism type (Theorem 7.1). 


\section{Preliminaries}

In this section we review certain aspects of simplicial complexes and their face rings that will be needed in the rest of the paper. An excellent general reference to this material is [30].

\subsection{Simplicial complexes}

Let $\Delta$ be a $(d-1)$-dimensional simplicial complex. The main object of our study is the $f$-vector of $\Delta, f(\Delta):=\left(f_{-1}(\Delta), f_{0}(\Delta), \ldots, f_{d-1}(\Delta)\right)$, where $f_{i}(\Delta)$ denotes the number of $i$-dimensional faces of $\Delta$. It is sometimes more convenient to work with the $h$-vector of $\Delta, h(\Delta)=\left(h_{0}(\Delta), \ldots, h_{d}(\Delta)\right)$, defined by

$$
\sum_{i=0}^{d} h_{i} x^{d-i}=\sum_{i=0}^{d} f_{i-1}(x-1)^{d-i} .
$$

Thus, $h_{0}(\Delta)=1, h_{1}(\Delta)=f_{0}(\Delta)-d$, and $h_{d}(\Delta)=(-1)^{d-1} \tilde{\chi}(\Delta)$, where $\tilde{\chi}(\Delta)$ is the reduced Euler characteristic of $\Delta$.

Fix an infinite field $k$. Another set of invariants we need is the set of the Betti numbers,

$$
\beta_{i}(\Delta):=\operatorname{dim}_{\mathrm{k}} \tilde{H}_{i}(\Delta ; \mathrm{k})=\operatorname{dim}_{\mathrm{k}} \tilde{H}_{i}(\|\Delta\| ; \mathrm{k}), \quad 0 \leq i \leq \operatorname{dim} \Delta .
$$

Here $\tilde{H}_{i}(\Delta ; \mathrm{k})$ is the $i$-th reduced simplicial homology of $\Delta$ computed with coefficients in $\mathrm{k},\|\Delta\|$ is the geometric realization of $\Delta$, and $\tilde{H}_{i}(\|\Delta\| ; \mathrm{k})$ is the $i$-th singular homology.

For a face $\tau$ of $\Delta$, the link of $\tau$ in $\Delta$ is lk $\tau:=\{\sigma: \tau \cap \sigma=\emptyset, \tau \cup \sigma \in \Delta\}$. We use the following terminology: $\Delta$ is a $\mathrm{k}$-Cohen-Macaulay complex (k-CM, for short) if for every face $\tau \in \Delta$ (including the empty face), $\beta_{i}(\mathrm{lk} \tau)=0$ for all $i<d-|\tau|-1$; if in addition $\beta_{d-|\tau|-1}(\mathrm{lk} \tau)=1$ for all $\tau \in \Delta$, then $\Delta$ is called a k-homology sphere. For instance, three triangles glued together along a common edge form a k-Cohen-Macaulay complex which is not a k-homology sphere. When no confusion is likely we may suppress the field $\mathrm{k}$.

A face of $\Delta$ is a facet if it is maximal under inclusion. We say that $\Delta$ is pure if all facets of $\Delta$ have dimension $d-1$. Following [8, Definition 1.1], we call $\Delta$ a pseudomanifold if it is pure and each codimension-one face of $\Delta$ is contained in exactly two facets. (Note that we do not assume that a pseudomanifold is strongly connected.)

A pure simplicial complex $\Delta$ is k-Buchsbaum if all vertex links of $\Delta$ are $\mathrm{k}-\mathrm{CM}$, and it is a k-homology manifold if all vertex links are k-homology spheres. A pure simplicial complex $\Delta$ is a $\mathrm{k}$-space with isolated singularities if all vertex links of $\Delta$ are k-Buchsbaum (equivalently, all edge links are $\mathrm{k}-\mathrm{CM}$ ), and it is a k-pseudomanifold with isolated singularities if all vertex links are 
k-homology manifolds (equivalently, all edge links are k-homology spheres). A vertex $v$ of a space (pseudomanifold, resp.) with isolated singularities is called singular if the link of $v$ is not k-CM (not a k-homology sphere, resp.).

A few comments are in order. Our definition of a space with isolated singularities is not typical as we allow the link of a singularity to be any Buchsbaum complex rather than a manifold. Note also that this definition implies that singularities can only occur at the vertices of the complex. Finally, observe that an elementary application of excision shows that whether or not $\Delta$ is a space with isolated singularities or a pseudomanifold with isolated singularities is a topological property of $\Delta$.

\subsection{Face rings}

The face ring (also known as the Stanley-Reisner ring) of a simplicial complex $\Delta$ on the vertex set $V$ is

$$
\mathrm{k}[\Delta]:=\mathrm{k}\left[x_{v}: v \in V\right] / I_{\Delta},
$$

where $I_{\Delta}$ is the squarefree monomial ideal generated by non-faces:

$$
I_{\Delta}=\left(x_{v_{1}} \ldots x_{v_{k}}:\left\{v_{1}, \ldots, v_{k}\right\} \notin \Delta\right) .
$$

It is a result of Reisner [23] that $\Delta$ is a k-CM simplicial complex if and only if $k[\Delta]$ is a Cohen-Macaulay ring, and it is a result of Schenzel [25] that $\Delta$ is a k-Buchsbaum complex if and only if $k[\Delta]$ is a Buchsbaum ring.

A linear system of parameters (an l.s.o.p., for short) for $k[\Delta]$ is a set of $d=\operatorname{dim} \Delta+1$ linear forms $\Theta=\left\{\theta_{1}, \ldots, \theta_{d}\right\}$ such that

$$
\mathrm{k}(\Delta, \Theta):=k[\Delta] /\left(\theta_{1}, \ldots, \theta_{d}\right)
$$

is a finite-dimensional $\mathrm{k}$-space. If $\mathrm{k}$ is an infinite field, then an 1.s.o.p. always exists. The ring $\mathrm{k}(\Delta, \Theta)$ is called an Artinian reduction of $\mathrm{k}[\Delta]$.

\subsection{Local cohomology}

Let $\mathfrak{m}=\left(x_{v}: v \in V\right)$ be the irrelevant ideal of the polynomial ring $S:=\mathrm{k}\left[x_{v}\right.$ : $v \in V]$. For a finitely-generated graded $S$-module $M$, we denote by $M_{k}$ the $k$-th homogeneous component of $M$ and by $H_{\mathfrak{m}}^{i}(M)$ the $i$-th local cohomology of $M$ with respect to $\mathfrak{m}$. For $t \in S$ define $\left(0:_{M} t\right):=\{v \in M: t v=0\}$. The socle of $M$ is

$$
\operatorname{Soc}(M)=\bigcap_{v \in V}\left(0:_{M} x_{v}\right)=\{v \in M: \mathfrak{m} v=0\} .
$$

For a simplicial complex $\Delta$, Gräbe [9] gave a description of $H_{\mathfrak{m}}^{i}(\mathrm{k}[\Delta])$ and its $S$-module structure in terms of the simplicial cohomology of the links of 
$\Delta$ and the maps between them. In the case when $\Delta$ is a space with isolated singularities, this description takes the following simple form that we review now. For $v \in V$, let costar of $v$ be $\operatorname{cost}(v)=\{\tau \in \Delta: v \notin \tau\}$, and consider the $i$-th simplicial cohomology of the pair $(\Delta, \operatorname{cost} v)$ with coefficients in $\mathrm{k}$ :

$$
H_{\{v\}}^{i}(\Delta):=H^{i}(\Delta, \operatorname{cost} v ; \mathrm{k}) \cong H^{i}(\|\Delta\|,\|\Delta\|-v ; \mathrm{k}) \cong \tilde{H}^{i-1}(\mathrm{lk} v ; \mathrm{k}) .
$$

Also set $H_{\emptyset}^{i}(\Delta):=H^{i}(\Delta, \emptyset ; \mathrm{k})=\tilde{H}^{i}(\Delta ; \mathrm{k})$, and let $\iota^{*}: H_{\{v\}}^{i}(\Delta) \rightarrow H_{\emptyset}^{i}(\Delta)$, for $v \in V$, be the map induced by inclusion. In the following, $\delta_{w v}$ denotes the Kronecker delta, and $\left(s_{v}\right)_{v \in V}$ an element of $\bigoplus_{v \in V} H_{\{v\}}^{i}(\Delta)$.

TheOREM 2.1 (Gräbe). Let $\Delta$ be a space with isolated singularities and $-1 \leq i<\operatorname{dim} \Delta$. Then

$$
H_{\mathfrak{m}}^{i+1}(\mathrm{k}[\Delta])_{-j}= \begin{cases}0 & \text { (if } j<0), \\ H_{\emptyset}^{i}(\Delta) & (\text { if } j=0), \\ \bigoplus_{v \in V} H_{\{v\}}^{i}(\Delta) & \text { (if } j>0) .\end{cases}
$$

The S-module structure on $H_{\mathfrak{m}}^{i+1}(\mathrm{k}[\Delta])$ is given by

$$
\begin{aligned}
x_{w}: H_{\mathfrak{m}}^{i+1}(\mathrm{k}[\Delta])_{-(j+1)} & \rightarrow H_{\mathfrak{m}}^{i+1}(\mathrm{k}[\Delta])_{-j} \\
\left(s_{v}\right)_{v \in V} & \mapsto \begin{cases}\iota^{*}\left(s_{w}\right) & \text { if } j=0, \\
\left(\delta_{w v} s_{v}\right)_{v \in V} & \text { if } j>0 .\end{cases}
\end{aligned}
$$

\section{Dehn-Sommerville relations}

One of the nicest properties of the $h$-vectors of homology manifolds is the Dehn-Sommerville relations due to Klee [13] asserting that if $\Gamma$ is an $(r-1)$ dimensional homology manifold, then

(1) $h_{r-i}(\Gamma)=h_{i}(\Gamma)+(-1)^{i-1}\left(\begin{array}{l}r \\ i\end{array}\right)\left(1+(-1)^{r} \tilde{\chi}(\Gamma)\right) \quad$ for all $0 \leq i \leq r$.

The goal of this section is to derive the following generalization of Eq. (1) for pseudomanifolds with isolated singularities. In principle this could be done using the results of [4] or [35, Lemma 2] that are stated in terms of $f$-vectors and are designed to include more general complexes. However, as we will require an $h$-vector version and only need it for pseudomanifolds with isolated singularities, we derive the formula ourselves. By convention, for all $a, b \in \mathrm{Z}$, $\left(\begin{array}{l}b \\ 0\end{array}\right)=1$, and $\left(\begin{array}{l}b \\ a\end{array}\right)=0$ if $a<0$. 
THEOREM 3.1. Let $\Delta$ be a $(d-1)$-dimensional simplicial complex on the vertex set $V$. If $\Delta$ is a pseudomanifold with isolated singularities, then for all $0 \leq i \leq d$,

$$
\begin{aligned}
h_{d-i}(\Delta)=h_{i}(\Delta)+(-1)^{i-1} & \left(\begin{array}{c}
d \\
i
\end{array}\right)\left(1+(-1)^{d} \tilde{\chi}(\Delta)\right) \\
& +(-1)^{i}\left(\begin{array}{c}
d-1 \\
i-1
\end{array}\right) \sum_{v \in V}\left(1+(-1)^{d-1} \tilde{\chi}(\mathrm{lk} v)\right) .
\end{aligned}
$$

Proof. We utilize Eq. (1) applied to the links of vertices - notice that since $\Delta$ is a pseudomanifold with isolated singularities, all vertex links are $(d-2)$ dimensional homology manifolds. Another ingredient needed for the proof is the following connection between the $h$-vector of a pure simplicial complex and the sums of the $h$-vectors of the vertex links (see [32, Proposition 2.3]):

(2) $\quad i h_{i}(\Delta)+(d-i+1) h_{i-1}(\Delta)=\sum_{v \in V} h_{i-1}(\mathrm{lk} v) \quad$ for all $1 \leq i \leq d$.

The proof of the theorem is by induction on $i$. For $i=0$ the statement reduces to

$$
h_{d}(\Delta)-h_{0}(\Delta)=-\left(1+(-1)^{d} \tilde{\chi}(\Delta)\right)
$$

This holds as $h_{0}(\Delta)=1$ and $h_{d}(\Delta)=(-1)^{d-1} \tilde{\chi}(\Delta)$ for all simplicial complexes.

For $i>0$, we have

$$
\begin{aligned}
i h_{i}(\Delta)+(d-i+1) h_{i-1}(\Delta) \\
=\sum_{v \in V} h_{i-1}(\mathrm{lk} v) \\
=\sum_{v \in V}\left[h_{(d-1)-(i-1)}(\mathrm{lk} v)+(-1)^{i-1}\left(\begin{array}{c}
d-1 \\
i-1
\end{array}\right)\left(1+(-1)^{d-1} \tilde{\chi}(\mathrm{lk} v)\right)\right] \\
=\left[\sum_{v \in V} h_{d-i}(\mathrm{lk} v)\right]+(-1)^{i-1}\left(\begin{array}{c}
d-1 \\
i-1
\end{array}\right) \sum_{v \in V}\left(1+(-1)^{d-1} \tilde{\chi}(\mathrm{lk} v)\right) \\
=(d-i+1) h_{d-i+1}(\Delta)+i h_{d-i}(\Delta) \\
\quad+(-1)^{i-1}\left(\begin{array}{c}
d-1 \\
i-1
\end{array}\right) \sum_{v \in V}\left(1+(-1)^{d-1} \tilde{\chi}(\mathrm{lk} v)\right),
\end{aligned}
$$


where the first and the last steps follow from Eq. (2), and the second step from Eq. (1) applied to the vertex links. Thus

$$
\begin{aligned}
i\left(h_{d-i}-h_{i}\right)=-(d-i & +1)\left(h_{d-(i-1)}-h_{i-1}\right) \\
& +(-1)^{i}\left(\begin{array}{c}
d-1 \\
i-1
\end{array}\right) \sum_{v \in V}\left(1+(-1)^{d-1} \tilde{\chi}(\operatorname{lk} v)\right) .
\end{aligned}
$$

Substituting in Eq. (3) our inductive hypothesis for $h_{d-(i-1)}-h_{i-1}$ and using that

$$
\frac{1}{i} \cdot(d-i+1)\left(\begin{array}{c}
d \\
i-1
\end{array}\right)=\left(\begin{array}{l}
d \\
i
\end{array}\right)
$$

and

$$
\frac{1}{i} \cdot\left[(d-i+1)\left(\begin{array}{c}
d-1 \\
i-2
\end{array}\right)+\left(\begin{array}{c}
d-1 \\
i-1
\end{array}\right)\right]=\left(\begin{array}{c}
d-1 \\
i-1
\end{array}\right)
$$

yields the result.

The above formula can be written exclusively in terms of $\chi(\Delta)$. When $d$ is odd every vertex link is an odd-dimensional homology manifold, so its Euler characteristic is zero and Theorem 3.1 reduces to Eq. (1). When $d$ is even, there exists $\Delta^{\prime}$-an odd-dimensional homology manifold with boundary whose boundary consists of the disjoint union of the vertex links of $\Delta-$ such that $\Delta$ is homeomorphic to $\Delta^{\prime}$ with each boundary component coned off. (To obtain such $\Delta^{\prime}$, consider the second barycentric subdivision of $\Delta$ and delete the open stars of singular vertices.) The Euler characteristic of $\Delta^{\prime}$ is one-half the Euler characteristic of its boundary and hence equals one-half the sum of the Euler characteristics of the vertex links and, of course, the Euler characteristic of each cone is one. Hence,

$$
\chi(\Delta)=\frac{1}{2} \sum_{v \in V} \chi(\mathrm{lk} v)+|V|-\sum_{v \in V} \chi(\mathrm{lk} v)
$$

and

$$
\sum_{v \in V}\left(1+(-1)^{d-1} \tilde{\chi}(\mathrm{lk} v)\right)=2|V|-\sum_{v \in V} \chi(\operatorname{lk} v)=2 \chi(\Delta) .
$$

\section{Homologically isolated singularities}

We now turn our attention to inequalities. In this section we derive lower bounds on the face numbers of spaces with homologically isolated singularities - a certain subclass of spaces with isolated singularities introduced in [18, Section 4]. To achieve this goal we first compute the Hilbert series of Artinian reductions of such complexes generalizing Schenzel's formula for Buchsbaum 
complexes [25], which in turn is a generalization of Stanley's formula for CM complexes [28].

We use the notation of Subsection 2.3. For a linear form $\theta=\sum_{v \in V} a_{v} x_{v}$ with all $a_{v} \neq 0$, consider the maps $\iota^{*}: H_{\{v\}}^{i}(\Delta) \rightarrow H_{\emptyset}^{i}(\Delta)$ and their weighted direct sum

$$
f^{i, \theta}:\left[\bigoplus_{v \in V} H_{\{v\}}^{i}(\Delta)\right] \rightarrow H_{\emptyset}^{i}(\Delta), \quad f^{i, \theta}=\sum_{v \in V} a_{v} \iota^{*}\left[H_{\{v\}}^{i}(\Delta) \rightarrow H_{\emptyset}^{i}(\Delta)\right] .
$$

Define $\mathscr{K}_{i}^{\theta}(\Delta):=\operatorname{dim}_{\mathrm{k}} \operatorname{ker} f^{i, \theta}$ and $\mathscr{C}_{i}^{\theta}(\Delta):=\operatorname{dim}_{\mathrm{k}} \operatorname{coker} f^{i, \theta}$.

Definition 4.1. We say that a $(d-1)$-dimensional simplicial complex $\Delta$ on the vertex set $V$ has homologically isolated singularities if (i) it is a space with isolated singularities, and (ii) for all $0 \leq i \leq d-2$, the images $\iota^{*}: H_{\{v\}}^{i}(\Delta) \rightarrow H_{\emptyset}^{i}(\Delta)$ for $v \in V$ are linearly independent subspaces of $H_{\emptyset}^{i}(\Delta)$. Equivalently, the kernel of the above map $f^{i, \theta}$ decomposes as a direct sum of the kernels of the summands, and in particular the dimension of the kernel is independent of a specific choice of $\theta$ (as long as all coefficients of $\theta$ are non-vanishing); in this case we write $\mathscr{K}_{i}$ and $\mathscr{C}_{i}$ instead of $\mathscr{K}_{i}^{\theta}$ and $\mathscr{C}_{i}^{\theta}$, resp.

Let $X=\|\Delta\|$, and let $\Sigma$ be the set of singularities of $X$. Since for a nonsingular vertex $v$ and $i \leq d-2, H_{\{v\}}^{i}(\Delta)=0$, and since $H_{\{v\}}^{i}(\Delta)$ is canonically isomorphic to $H^{i}(X, X-v ; \mathrm{k})$ while $H_{\emptyset}^{i}(\Delta)$ is canonically isomorphic to $\tilde{H}^{i}(X ; \mathrm{k})$, it follows that $\Delta$ has homologically isolated singularities if and only if for all $0 \leq i \leq d-2$, the images $\iota^{*}: H^{i}(X, X-v ; \mathrm{k}) \rightarrow \tilde{H}^{i}(X ; \mathrm{k})$ for $v \in \Sigma$ are linearly independent subspaces of $\tilde{H}^{i}(X ; \mathrm{k})$. Thus being a pseudomanifold with homologically isolated singularities is a topological invariant.

Evidently, any complex with only one isolated singularity has homologically isolated singularities. Many other examples of such complexes are described in [18, Section 4]. On the other hand, suspensions of manifolds have isolated singularities that are not homologically isolated.

Schenzel [25] proved that if $\Delta$ is a $\mathrm{k}$-Buchsbaum complex of dimension $d-1$, then the Hilbert series of $\mathrm{k}(\Delta, \Theta)$ is independent of the choice of an 1.s.o.p. $\Theta$ and is given by

$$
F(\mathrm{k}(\Delta, \Theta), \lambda)=\sum_{i=0}^{d}\left(h_{i}(\Delta)+\left(\begin{array}{l}
d \\
i
\end{array}\right) \sum_{j=1}^{i-1}(-1)^{i-j-1} \beta_{j-1}(\Delta)\right) \lambda^{i} .
$$

If $\Delta$ is k-CM, this formula reduces to Stanley's result: $F(\mathrm{k}(\Delta, \Theta), \lambda)=$ $\sum_{i=0}^{d} h_{i} \lambda^{i}$. Here (see Theorems 4.2 and 4.7) we extend Schenzel's result to all complexes with homologically isolated singularities. The main point is that 
the Hilbert series of an Artinian reduction of the face ring of such a complex $\Delta$ is determined by the $f$-numbers and the homeomorphism type of $\Delta$.

THEOREM 4.2. Let $\Delta$ be a $(d-1)$-dimensional simplicial complex with homologically isolated singularities. Then for any l.s.o.p. $\Theta$ of $\mathrm{k}[\Delta]$, the Hilbert series of $\mathrm{k}(\Delta, \Theta)$ is equal to $\sum_{i=0}^{d} h_{i}^{\prime}(\Delta) \lambda^{i}$, where (for $0 \leq i \leq d$ )

$$
\begin{aligned}
h_{i}^{\prime}(\Delta)=h_{i}(\Delta)+ & \left(\begin{array}{c}
d \\
i
\end{array}\right) \sum_{j=1}^{i-1}(-1)^{i-j-1} \beta_{j-1}(\Delta) \\
& +\left(\begin{array}{c}
d-1 \\
i
\end{array}\right)\left[\mathscr{K}_{i-1}(\Delta)-\sum_{v \in V} \sum_{j=1}^{i-2}(-1)^{i-j} \beta_{j-1}(\mathrm{lk} v)\right] .
\end{aligned}
$$

The crucial part of the proof is the following property of the face rings of complexes with homologically isolated singularities that was observed in [18, Section 4].

Lemma 4.3. Let $\Delta$ be a $(d-1)$-dimensional simplicial complex with isolated singularities and let $\theta=\sum_{v \in V} a_{v} x_{v}$ be a 1-form with all coefficients nonvanishing. Then

(1) For all $i<d-1$, the Hilbert series of the $i$-th local cohomology of $\mathrm{k}[\Delta] /(\theta), F\left(H_{\mathfrak{m}}^{i}(\mathrm{k}[\Delta] /(\theta)), \lambda\right)$, equals $\beta_{i}(\Delta) \lambda+\left(\mathscr{K}_{i}^{\theta}(\Delta)+\mathscr{C}_{i-1}^{\theta}(\Delta)\right)$.

(2) If $\Delta$ has homologically isolated singularities, then $\mathrm{k}[\Delta] /(\theta)$ is a Buchsbaum $S$-module.

Proof. (Sketch) The first part follows from Theorem 2.1 together with the long exact sequence in local cohomology arising from the short exact sequence

$$
0 \rightarrow \mathrm{k}[\Delta] \stackrel{\cdot \theta}{\longrightarrow} \mathrm{k}[\Delta] \rightarrow \mathrm{k}[\Delta] /(\theta) \rightarrow 0 .
$$

Moreover, the condition on homologically isolated singularities implies that

$$
\mathfrak{m} H_{\mathfrak{m}}^{i}(\mathrm{k}[\Delta] /(\theta))=0 .
$$

The second part then follows from [31, Prop. I.3.10].

A comprehensive reference to Buchsbaum rings and modules is [31]. Here we will need only a few standard properties that we record in the following lemma.

Lemma 4.4. Let $\Delta$ be a $(d-1)$-dimensional simplicial complex with homologically isolated singularities and let $\theta_{1}, \ldots, \theta_{d}$ be an l.s.o.p. for $\mathrm{k}[\Delta]$ with $\theta_{1}$ having non-vanishing coefficients. Then for all $1 \leq s \leq d-1$, 
(1) $\mathrm{k}[\Delta]\{s\}:=\mathrm{k}[\Delta] /\left(\theta_{1}, \ldots, \theta_{s}\right)$ is a Buchsbaum $S$-module of Krull dimension $d-s$,

(2) $\left(0::_{\mathrm{k}[\Delta]\{s\}} \theta_{s+1}\right)=H_{\mathfrak{m}}^{0}(\mathrm{k}[\Delta]\{s\})$, and

(3) for all $i+s<d-1$ and $j \in \mathbf{Z}$,

$$
H_{\mathfrak{m}}^{i}(\mathrm{k}[\Delta]\{s+1\})_{j} \cong H_{\mathfrak{m}}^{i}(\mathrm{k}[\Delta]\{s\})_{j} \oplus H_{\mathfrak{m}}^{i+1}(\mathrm{k}[\Delta]\{s\})_{j-1} .
$$

Proof. Part 1 is a consequence of Lemma 4.3(2) above and Corollary 1.11 on p. 65 of [31]; Part 2 follows from Part 1 and Proposition 1.10 on p. 64 of [31]; Part 3 is immediately apparent from Part 1 and [20, Lemma 8.2].

Lemma 4.5. For $\Delta$ and $\theta_{1}, \ldots, \theta_{d}$ as in Lemma 4.4, and for all $s \geq 1$, $i+s<d$, and $j \in \mathrm{Z}$,

$$
\begin{aligned}
\operatorname{dim}_{\mathrm{k}} H_{\mathrm{m}}^{i}(\mathrm{k}[\Delta]\{s\})_{j}=\left(\begin{array}{c}
s-1 \\
j-1
\end{array}\right) & \beta_{i+j-1}(\Delta) \\
& +\left(\begin{array}{c}
s-1 \\
j
\end{array}\right)\left(\mathscr{K}_{i+j}(\Delta)+\mathscr{C}_{i+j-1}(\Delta)\right) .
\end{aligned}
$$

In particular, for $1 \leq s \leq d-1$ and $j \in \mathbf{Z}$,

$$
\begin{aligned}
\operatorname{dim}_{\mathrm{k}}(0: \mathrm{k}[\Delta]\{s\} & \left.\theta_{s+1}\right)_{j}=\left(\begin{array}{c}
s-1 \\
j-1
\end{array}\right) \beta_{j-1}(\Delta) \\
& +\left(\begin{array}{c}
s-1 \\
j
\end{array}\right)\left(\mathscr{K}_{j}(\Delta)+\mathscr{C}_{j-1}(\Delta)\right) .
\end{aligned}
$$

Proof. We use induction on $s$. For $s=1$ the statement reduces to Part 1 of Lemma 4.3. Part 3 of Lemma 4.4 together with the binomial identity $\left(\begin{array}{l}a \\ b\end{array}\right)+$ $\left(\begin{array}{c}a \\ b-1\end{array}\right)=\left(\begin{array}{c}a+1 \\ b\end{array}\right)$ gives the inductive step. The "in particular"-part then follows from Lemma 4.4(2).

We are now in a position to prove Theorem 4.2.

Proof. Since for any l.s.o.p. $\Theta=\left\{\theta_{1}, \ldots, \theta_{d}\right\}$ of $k[\Delta]$, the linear span of $\Theta$ contains a 1-form with all coefficients non-vanishing, we can assume w.l.o.g. that $\theta_{1}$ is such a form. It is a well-known fact (see for instance [31, p. 150]) that for a $(d-1)$-dimensional complex $\Delta$ and an 1.s.o.p. $\Theta$,

$$
F(\mathrm{k}(\Delta, \Theta), \lambda)=\sum_{i=0}^{d} h_{i}(\Delta) \lambda^{i}+\sum_{s=1}^{d-1} \lambda(1-\lambda)^{d-s-1} \cdot F\left(0:_{\mathrm{k}[\Delta]\{s\}} \theta_{s+1}, \lambda\right) .
$$


Substituting in this equation the "in particular"-part of Lemma 4.5 yields

$$
\begin{aligned}
& F(\mathrm{k}(\Delta, \Theta), \lambda)= \sum_{i=0}^{d} h_{i}(\Delta) \lambda^{i} \\
&+\sum_{s=1}^{d-1}(1-\lambda)^{d-s-1} \sum_{j=1}^{s} \lambda^{j+1}\left[\left(\begin{array}{c}
s-1 \\
j-1
\end{array}\right) \beta_{j-1}(\Delta)\right. \\
&\left.+\left(\begin{array}{c}
s-1 \\
j
\end{array}\right)\left(\mathscr{K}_{j}(\Delta)+\mathscr{C}_{j-1}(\Delta)\right)\right] .
\end{aligned}
$$

Comparing the coefficients of $\lambda^{i}$ on both sides we obtain

$$
\begin{aligned}
h_{i}^{\prime}(\Delta) & =h_{i}(\Delta)+\sum_{j=1}^{i-1}(-1)^{i-j-1}\left(\sum_{s=j}^{d-1}\left(\begin{array}{c}
d-s-1 \\
i-j-1
\end{array}\right)\left(\begin{array}{c}
s-1 \\
j-1
\end{array}\right)\right) \beta_{j-1}(\Delta) \\
& +\sum_{j=1}^{i-1}(-1)^{i-j-1}\left(\sum_{s=j+1}^{d-1}\left(\begin{array}{c}
d-s-1 \\
i-j-1
\end{array}\right)\left(\begin{array}{c}
s-1 \\
j
\end{array}\right)\right)\left(\mathscr{K}_{j}(\Delta)+\mathscr{C}_{j-1}(\Delta)\right) .
\end{aligned}
$$

Using that $\sum_{r=0}^{l}\left(\begin{array}{c}l-r \\ i-j\end{array}\right)\left(\begin{array}{l}r \\ j\end{array}\right)=\left(\begin{array}{c}l+1 \\ i+1\end{array}\right)$ then implies

$$
\begin{aligned}
h_{i}^{\prime}(\Delta)=h_{i}(\Delta)+\sum_{j=1}^{i-1}(-1)^{i-j-1} & {\left[\left(\begin{array}{c}
d-1 \\
i-1
\end{array}\right) \beta_{j-1}(\Delta)\right.} \\
& \left.+\left(\begin{array}{c}
d-1 \\
i
\end{array}\right)\left(\mathscr{K}_{j}(\Delta)+\mathscr{C}_{j-1}(\Delta)\right)\right] .
\end{aligned}
$$

Finally, it follows from exactness of

$$
0 \rightarrow \operatorname{ker}\left(f^{j, \theta}\right) \rightarrow \bigoplus_{v \in V} H_{\{v\}}^{j}(\Delta) \stackrel{f^{j, \theta}}{\longrightarrow} H_{\emptyset}^{j}(\Delta) \rightarrow \operatorname{coker}\left(f^{j, \theta}\right) \rightarrow 0
$$

that

$$
\mathscr{C}_{j}(\Delta)=\mathscr{K}_{j}(\Delta)+\beta_{j}(\Delta)-\sum_{v} \beta_{j-1}(\mathrm{lk} v) .
$$

We infer the theorem by substituting these expressions in Eq. (5) and using the identity $\left(\begin{array}{c}d-1 \\ i-1\end{array}\right)+\left(\begin{array}{c}d-1 \\ i\end{array}\right)=\left(\begin{array}{c}d \\ i\end{array}\right)$.

COROllary 4.6. Let $\Delta$ be a $(d-1)$-dimensional simplicial complex with homologically isolated singularities, and let $\Theta=\left\{\theta_{1}, \ldots, \theta_{d}\right\}$ be an l.s.o.p.for 
$\mathrm{k}[\Delta]$. Then for all $i<d-1$,

$$
\begin{aligned}
\operatorname{dim} \operatorname{Soc}(\mathrm{k}(\Delta, \Theta))_{i} \geq\left(\begin{array}{l}
d \\
i
\end{array}\right) \beta_{i-1}(\Delta)+\left(\begin{array}{c}
d-1 \\
i
\end{array}\right)\left(\mathscr{K}_{i}(\Delta)\right. \\
\left.+\mathscr{K}_{i-1}(\Delta)-\sum_{v \in V} \beta_{i-2}(\operatorname{lk} v)\right),
\end{aligned}
$$

and hence $h_{i}^{\prime}(\Delta) \geq\left(\begin{array}{c}d \\ i\end{array}\right) \beta_{i-1}(\Delta)+\left(\begin{array}{c}d-1 \\ i\end{array}\right)\left(\mathscr{K}_{i}(\Delta)+\mathscr{K}_{i-1}(\Delta)-\sum_{v \in V} \beta_{i-2}(\mathrm{lk} v)\right)$.

Proof. Since by Lemma 4.3(2), $\mathrm{k}[\Delta] /\left(\theta_{1}\right)$ is a Buchsbaum ring of Krull dimension $d-1$, Theorem 2.2 of [22] applies and gives

$$
\operatorname{dim} \operatorname{Soc}(\mathrm{k}(\Delta, \Theta))_{i} \geq \sum_{j=0}^{d-2}\left(\begin{array}{c}
d-1 \\
j
\end{array}\right) \operatorname{dim}_{\mathrm{k}} H_{\mathfrak{m}}^{j}\left(\mathrm{k}[\Delta] /\left(\theta_{1}\right)\right)_{i-j}
$$

By Lemma 4.3(1), for $i<d-1$ this inequality can be rewritten as

$$
\text { (7) } \begin{array}{r}
\operatorname{dim} \operatorname{Soc}(\mathrm{k}(\Delta, \Theta))_{i} \geq\left(\begin{array}{c}
d-1 \\
i-1
\end{array}\right) \beta_{i-1}(\Delta) \\
+\left(\begin{array}{c}
d-1 \\
i
\end{array}\right)\left(\mathscr{K}_{i}(\Delta)+\mathscr{C}_{i-1}(\Delta)\right) .
\end{array}
$$

The result follows from Eq. (6).

If $\Delta$ is a Buchsbaum complex, then for $i<d-1$ and $v \in V, \beta_{i-1}(\operatorname{lk} v)=$ $\mathscr{K}_{i}(\Delta)=0$. Thus, in this case, Theorem 4.2 reduces to Schenzel's theorem and Corollary 4.6 to [22, Theorem 3.4]. Theorem 4.2 and Corollary 4.6 can be restated in terms of singular homology.

THEOREM 4.7. Let $\Delta$ be $a(d-1)$-dimensional simplicial complex with isolated singularities, $X=\|\Delta\|$ its geometric realization, $\Sigma \subset X$ the set of singularities of $X$, and $\theta=\sum_{v \in V} x_{v}$. Then for all $j<d, \mathscr{K}_{j}^{\theta}(\Delta)+\mathscr{C}_{j-1}^{\theta}(\Delta)=$ $\operatorname{dim}_{\mathrm{k}} \tilde{H}_{j-1}(X-\Sigma ; \mathrm{k})$. Moreover, if $\Delta$ has homologically isolated singularities and $\Theta$ is any l.s.o.p. for $\mathrm{k}[\Delta]$, then for $0 \leq i \leq d$,

$$
\begin{aligned}
h_{i}^{\prime}(\Delta)=h_{i}(\Delta)+\sum_{j=1}^{i-1}(-1)^{i-j-1}[ & \left(\begin{array}{c}
d-1 \\
i-1
\end{array}\right) \operatorname{dim}_{\mathrm{k}} \tilde{H}_{j-1}(X ; \mathrm{k}) \\
& \left.+\left(\begin{array}{c}
d-1 \\
i
\end{array}\right) \operatorname{dim}_{\mathrm{k}} \tilde{H}_{j-1}(X-\Sigma ; \mathrm{k})\right],
\end{aligned}
$$


and for $i<d-1$,

$$
\begin{aligned}
\operatorname{dim} \operatorname{Soc}(\mathrm{k}(\Delta, \Theta))_{i} \geq\left(\begin{array}{c}
d-1 \\
i-1
\end{array}\right) \operatorname{dim}_{\mathrm{k}} & \tilde{H}_{i-1}(X ; \mathrm{k}) \\
& +\left(\begin{array}{c}
d-1 \\
i
\end{array}\right) \operatorname{dim}_{\mathrm{k}} \tilde{H}_{i-1}(X-\Sigma ; \mathrm{k}) .
\end{aligned}
$$

Proof. The second assertion follows the first one and Eqs. (5) and (7), respectively. To prove the first assertion consider the following long cohomology sequence of the pair (where we omit $k$ to simplify the notation)

$$
\begin{aligned}
& H^{j-1}(X, X-\Sigma) \stackrel{\psi}{\longrightarrow} \tilde{H}^{j-1}(X) \\
& \rightarrow \tilde{H}^{j-1}(X-\Sigma) \rightarrow H^{j}(X, X-\Sigma) \stackrel{\phi}{\longrightarrow} \tilde{H}^{j}(X) .
\end{aligned}
$$

It implies that

$\operatorname{dim}_{\mathrm{k}} \tilde{H}_{j-1}(X-\Sigma)=\operatorname{dim}_{\mathrm{k}} \tilde{H}^{j-1}(X-\Sigma)=\operatorname{dim}_{\mathrm{k}} \operatorname{coker}(\psi)+\operatorname{dim}_{\mathrm{k}} \operatorname{ker}(\phi)$.

Thus to derive the first assertion it suffices to show that $\operatorname{dim}_{\mathrm{k}} \operatorname{coker}(\psi)=$ $\mathscr{C}_{j-1}^{\theta}(\Delta)$ and $\operatorname{dim}_{\mathrm{k}} \operatorname{ker}(\phi)=\mathscr{K}_{j}^{\theta}(\Delta)$. This is immediate from the following series of canonical isomorphisms:

$$
\begin{aligned}
H^{j}(X, X-\Sigma) & \cong \bigoplus_{v \in \Sigma} H^{j}(X, X-v) \\
& \cong \bigoplus_{v \in V} H^{j}(X, X-v) \\
& \cong \bigoplus_{v \in V} H_{\{v\}}^{j}(\Delta) \quad \forall j<d-1 .
\end{aligned}
$$

The first isomorphism in this series follows, for instance, by induction on the size of $\Sigma$ using the relative form of the Mayer-Vietoris sequence for the inductive step. The last isomorphism is implied by the fact that $\|\operatorname{cost} v\|$ is a deformation retract of $X-v$. The middle one is immediate from the definition of a non-singular vertex: for such a vertex $v$ and $j<d-1, H^{j}(X, X-v) \cong$ $\tilde{H}^{j-1}(\mathrm{lk} v)=0$.

Remark 4.8. When $X=\|\Delta\|$ is a pseudomanifold with isolated singularities and $j<\operatorname{dim} X, \tilde{H}_{j-1}(X-\Sigma ; \mathrm{k})$ coincides with $H_{j-1}^{(\overline{0})}(X ; \mathrm{k})-$ the $(j-1)$-st intersection homology of perversity $\overline{0}$. (Intersection homology was introduced and studied in [8].) Thus for pseudomanifolds with homologically isolated singularities, $h_{i}^{\prime}(\Delta)-h(\Delta)_{i}$ can be written as a linear combination of the usual Betti numbers of $\Delta$ and the intersection Betti numbers of $\|\Delta\|$. 
We now turn to numerical consequences of Theorem 4.2. Let

$$
g_{2}(\Delta):=h_{2}(\Delta)-h_{1}(\Delta) .
$$

The celebrated Lower Bound Theorem [3], [12] asserts that if $\Delta$ is a connected k-homology manifold of dimension $d-1 \geq 2$, then $g_{2}(\Delta) \geq 0$. Moreover, it was shown in [22, Theorem 5.3] that if $\Delta$ is a connected orientable khomology manifold of dimension $d-1 \geq 3$, then $g_{2}(\Delta) \geq\left(\begin{array}{c}d+1 \\ 2\end{array}\right) \beta_{1}(\Delta)$. Here we extend this result to pseudomanifolds with homologically isolated singularities. (For normal pseudomanifolds, that is, pseudomanifolds all of whose faces of codimension at least two have connected links, it is a result of Fogelsanger [7] that the lower bound $g_{2} \geq 0$ continues to hold.)

THEOREM 4.9. Let $\Delta$ be a $(d-1)$-dimensional pseudomanifold with homologically isolated singularities. If $d \geq 5$, then

$$
\begin{aligned}
g_{2}(\Delta) \geq\left(\begin{array}{c}
d+1 \\
2
\end{array}\right) & {\left[\beta_{d-2}(\Delta)-\beta_{d-1}(\Delta)+1\right] } \\
& +d \cdot \mathscr{K}_{d-2}(\Delta)-d \sum_{v \in V}\left[\beta_{d-3}(\operatorname{lk} v)-\beta_{d-2}(\operatorname{lk} v)+1\right] .
\end{aligned}
$$

The same assertion also holds if $d=4$ and $\Delta$ has at most 5 singularities.

If $\Delta$ is a connected orientable k-homology manifold, then $\beta_{d-1}(\Delta)=1$ and by Poincaré duality $\beta_{d-2}(\Delta)=\beta_{1}(\Delta)$; also for every vertex $v, \beta_{d-2}(\mathrm{lk} v)=1$ while $\beta_{d-3}(\mathrm{lk} v)=0$. Thus in this case Theorem 4.9 is equivalent to the inequality $g_{2}(\Delta) \geq\left(\begin{array}{c}d+1 \\ 2\end{array}\right) \beta_{1}(\Delta)$ mentioned above. In general the right-hand side is a topological invariant of $\Delta$ as all of the nonsingular vertices contribute zero to the last term.

Proof. First assume $d \geq 5$. Since $\Delta$ is a $(d-1)$-dimensional pseudomanifold with isolated singularities, it follows that the link of every $(d-4)$-face $F$ is a 2-dimensional homology sphere, and hence the boundary of a 3-dimensional simplicial polytope. Thus for a generic 1-form $\omega$, multiplication by $\omega$ considered as a linear map from $\mathrm{k}(\mathrm{lk} F)_{1}$ to $\mathrm{k}(\mathrm{lk} F)_{2}$, where $\mathrm{k}(\mathrm{lk} F)$ is an Artinian reduction of $\mathrm{k}[\mathrm{k} F]$ by a generic 1.s.o.p., is an isomorphism. (In characteristic 0 this result was proved in [29]; for a field of arbitrary characteristic it follows from Murai's paper [19] combined with Whiteley's proof that 2-dimensional spheres are strongly edge decomposable [36].) The proof of Theorem 4.26 in [33] then implies that if $\Theta$ is a generic 1.s.o.p. of $k[\Delta]$ and $\omega \in \mathrm{k}[\Delta]$ is a generic 1-form, then the map

$$
\cdot \omega: \mathrm{k}(\Delta, \Theta)_{d-2} \rightarrow \mathrm{k}(\Delta, \Theta)_{d-1},
$$


is surjective. As the socle, $\operatorname{Soc}(\mathrm{k}(\Delta, \Theta))_{d-2}$, is in the kernel of this map, we obtain that

$$
h_{d-2}^{\prime}(\Delta)-\operatorname{dim}_{\mathrm{k}} \operatorname{Soc}(\mathrm{k}(\Delta, \Theta))_{d-2} \geq h_{d-1}^{\prime}(\Delta) .
$$

If $d=4$ and $\Delta$ has at most 5 singular vertices, then we appeal to Lemma 6.2 in Section 6 below to see that Eq. (8) holds in this case as well.

Since $\Delta$ has homologically isolated singularities, Theorem 4.2 implies that

$$
\begin{aligned}
& h_{d-1}^{\prime}(\Delta)=h_{d-1}(\Delta)+d\left[\sum_{j=1}^{d-2}(-1)^{(d-1)-j-1} \beta_{j-1}(\Delta)\right] \\
& +\mathscr{K}_{d-2}(\Delta)-\sum_{v \in V} \sum_{j=1}^{d-3}(-1)^{(d-1)-j} \beta_{j-1}(\mathrm{lk} v) \\
& =h_{d-1}(\Delta)+d\left[(-1)^{d-1} \tilde{\chi}(\Delta)-\beta_{d-1}(\Delta)+\beta_{d-2}(\Delta)\right] \\
& \quad+\mathscr{K}_{d-2}(\Delta)-\sum_{v \in V}\left[(-1)^{d} \tilde{\chi}(\mathrm{lk} v)-\beta_{d-2}(\mathrm{lk} v)+\beta_{d-3}(\mathrm{lk} v)\right] \\
& =h_{1}(\Delta)+d\left[\beta_{d-2}(\Delta)-\beta_{d-1}(\Delta)+1\right] \\
& \quad+\mathscr{K}_{d-2}(\Delta)-\sum_{v \in V}\left[\beta_{d-3}(\mathrm{lk} v)-\beta_{d-2}(\mathrm{lk} v)+1\right],
\end{aligned}
$$

where the last step follows from Theorem 3.1. A similar computation using Theorems 4.2 and 3.1, and Corollary 4.6 gives

$$
\begin{aligned}
& h_{d-2}^{\prime}(\Delta)-\operatorname{dim}_{\mathrm{k}} \operatorname{Soc}(\mathrm{k}(\Delta, \Theta))_{d-2} \\
& \leq h_{2}-\left(\begin{array}{l}
d \\
2
\end{array}\right)\left[\beta_{d-2}(\Delta)-\beta_{d-1}(\Delta)+1\right]-(d-1) \mathscr{K}_{d-2}(\Delta) \\
& +(d-1) \sum_{v \in V}\left[\beta_{d-3}(\operatorname{lk} v)-\beta_{d-2}(\operatorname{lk} v)+1\right] .
\end{aligned}
$$

Substituting Eqs. (9) and (10) in (8) and combining like terms yields the result.

A $(d-1)$-dimensional simplicial complex $\Delta$ is called Eulerian if for every face $\tau \in \Delta$ (including the empty face), the link of $\tau$ has the same Euler characteristic as the sphere of dimension $d-1-|\tau|$. Another famous conjecture concerning the $f$-numbers is the Upper Bound Conjecture (UBC, for short). This conjecture, proposed for polytopes by Motzkin and for Eulerian complexes by Klee [14], asserts that in the class of all Eulerian simplicial complexes of dimension $d-1$ and with $n$ vertices, the boundary complex of a certain polytope (called the cyclic polytope) simultaneously maximizes all 
the $h$-numbers, and hence also all the $f$-numbers. Numerically, the UBC says that if $\Delta$ is an Eulerian $(d-1)$-dimensional complex with $n$ vertices, then

$$
h_{i}(\Delta) \leq\left(\begin{array}{c}
n-d+i-1 \\
i
\end{array}\right), \quad \text { for all } i \leq d / 2 .
$$

(It is a result of Klee that Eulerian complexes have symmetric $h$-vector: $h_{i}=$ $h_{d-i}$ for all $i[13]$.) The UBC is known to hold for all Eulerian complexes with $\geq O\left(d^{2}\right)$ vertices [14], as well as for all polytopes [17], homology spheres [27], and Eulerian homology manifolds [20]. In addition, a weaker version of the UBC (the one concerning the $f$-numbers) was verified in [11] to hold for all odd-dimensional Eulerian pseudomanifolds with isolated singularities. However the $h$-version of the UBC as well as even-dimensional cases remained open. Here we partially solve this problem by proving the following result.

THEOREM 4.10. Let $\Delta$ be a $(d-1)$-dimensional pseudomanifold with homologically isolated singularities. If $\Delta$ is Eulerian and has $n \geq 3 d-4$ vertices, then $h_{i}(\Delta) \leq\left(\begin{array}{c}n-d+i-1 \\ i\end{array}\right)$ for all $i \leq d / 2$.

The proof relies on Theorem 4.7 but otherwise is very similar to that given in [20], so we only sketch the main ideas, leaving details to our readers. To simplify notation, we write $\beta_{i}$ for $\operatorname{dim} \tilde{H}_{i}(X ; \mathrm{k}), \beta_{i}^{0}$ for $\operatorname{dim} \tilde{H}_{i}(X-\Sigma ; \mathrm{k})$, and $N_{i}$ for $\left(\begin{array}{c}n-d+i-1 \\ i\end{array}\right)$. We also write $h_{i}$ and $h_{i}^{\prime}$ instead of $h_{i}(\Delta)$ and $h_{i}^{\prime}(\Delta)$, respectively.

Proof. (Sketch) Since, for an arbitrary $j, \operatorname{Soc}(k(\Delta, \Theta))_{j}$ is an ideal of $\mathrm{k}(\Delta, \Theta)$, we have that

$$
A(\Delta, \Theta, j):=\mathrm{k}(\Delta, \Theta) /\left(\operatorname{Soc}(\mathrm{k}(\Delta, \Theta))_{j}\right)
$$

is a standard graded k-algebra. Hence the dimensions of the homogeneous components of $A(\Delta, \Theta, j)$ satisfy Macaulay's inequalities [30, p. 56]. Theorem 4.7 expresses these dimensions in terms of the $h$-numbers and $\beta, \beta^{0}$ numbers. Using these expressions together with Macaulay's conditions, a computation analogous to that in [20, Lemma 5.2] implies

$$
h_{j+1}^{\prime} \leq N_{j+1}-\sum_{i=1}^{j} \frac{N_{j+1}}{N_{i}}\left[\left(\begin{array}{c}
d-1 \\
i-1
\end{array}\right) \beta_{i-1}+\left(\begin{array}{c}
d-1 \\
i
\end{array}\right) \beta_{i-1}^{0}\right] \text { for all } j .
$$

Another rather straightforward computation shows that if $n \geq 3 d-4$, then

$$
\frac{N_{j+1}}{N_{i}} \cdot\left(\begin{array}{c}
d-1 \\
i-1
\end{array}\right) \geq\left(\begin{array}{c}
d-1 \\
j
\end{array}\right) \text { and } \frac{N_{j+1}}{N_{i}} \cdot\left(\begin{array}{c}
d-1 \\
i
\end{array}\right) \geq\left(\begin{array}{c}
d-1 \\
j+1
\end{array}\right)
$$

for all $1 \leq i \leq j-1 \leq \frac{d}{2}-2$. 
Substituting these inequalities in Eq. (11) yields $h_{j+1}^{\prime} \leq N_{j+1}-\sum_{i=1}^{j}\left[\left(\begin{array}{c}d-1 \\ j\end{array}\right) \beta_{i-1}+\left(\begin{array}{c}d-1 \\ j+1\end{array}\right) \beta_{i-1}^{0}\right] \quad$ for all $j \leq \frac{d}{2}-1$.

One last application of the formula from Theorem 4.7 relating $h_{j+1}$ to $h_{j+1}^{\prime}$ and Betti numbers then shows that $h_{j+1} \leq N_{j+1}$ for all $j+1 \leq d / 2$, as required.

\section{Depth $d-1$}

The depth of $\Delta$ is the largest $j$ such that the $(j-1)$-th skeleton of $\Delta$ is CM. For a space with isolated singularities this is equivalent to $\tilde{H}_{i}(\Delta ; \mathrm{k})=0$ for $i<j-1$ and for every vertex $v, \tilde{H}_{i}(\mathrm{lk} v ; \mathrm{k}) \cong H_{i+1}(\|\Delta\|,\|\Delta\|-v)=0$ for $i<j-2$; in particular, for a space with isolated singularities, having depth $j$ is a topological property. This notion coincides with the usual algebraic notion of depth for $\mathrm{k}[\Delta]$, see [26]. Complexes of dimension $d-1$ and depth $d-1$ are also known in the literature as almost $C M$ complexes.

Theorem 5.1. Let $\Delta$ be $a(d-1)$-dimensional complex with isolated singularities with depth $d-1$. Set $h_{i}^{\prime}=\operatorname{dim}_{\mathrm{k}} \mathrm{k}(\Delta, \Theta)_{i}$. Then for sufficiently generic $\Theta$

$$
\begin{aligned}
h_{d}^{\prime} & =\beta_{d-1}(\Delta) \\
h_{d-1}^{\prime} & =h_{d-1}(\Delta)+\operatorname{dim}_{\mathrm{k}}\left(\mathscr{K}_{d-2}^{\theta_{1}}(\Delta) \cap \cdots \cap \mathscr{K}_{d-2}^{\theta_{d}}(\Delta)\right) \\
h_{i}^{\prime} & =h_{i}(\Delta), \quad i<d-1 .
\end{aligned}
$$

Proof. For any $(d-1)$-dimensional simplicial complex $h_{d}^{\prime}=\beta_{d-1}$ for sufficiently generic $\Theta$, see [2, Lemma 2.2(3)]. Since $\Delta$ has depth $d-1$ the only possible nontrivial Betti numbers of $\Delta$ are $\beta_{d-1}$ and $\beta_{d-2}$ and the only potential nonzero Betti numbers for the link of every vertex $v$ are $\beta_{d-2}(\mathrm{lk} v)$ and $\beta_{d-3}(\mathrm{lk} v)$. By Theorem 2.1, $H_{\mathfrak{m}}^{i}(\mathrm{k}[\Delta])=0$ for $i<d-1$ and

$$
\begin{aligned}
H_{\mathrm{m}}^{d-1}(\mathrm{k}[\Delta])_{0} & \cong H_{\emptyset}^{d-2}(\Delta) \\
H_{\mathrm{m}}^{d-1}(\mathrm{k}[\Delta])_{-1} & \cong \sum_{v} H_{\{v\}}^{d-2}(\Delta) .
\end{aligned}
$$

Since $H_{\mathfrak{m}}^{0}(\mathrm{k}[\Delta])=0$, a prime avoidance argument (see, for instance, [6, Chapter 3]) implies the existence of $\theta_{1} \in \mathrm{k}[\Delta]_{1}$ such that multiplication by $\theta_{1}$ on $k[\Delta]$ is an injection. Now apply the induced long exact sequence for local cohomology with respect to the short exact sequence

$$
0 \rightarrow \mathrm{k}[\Delta] \stackrel{\cdot \theta_{1}}{\longrightarrow} \mathrm{k}[\Delta] \rightarrow \mathrm{k}[\Delta] /\left(\theta_{1}\right) \rightarrow 0
$$


to see that $H_{\mathrm{m}}^{i}\left(\mathrm{k}[\Delta] /\left(\theta_{1}\right)\right)=0$ for $i<d-2$. In addition, the module structure of $H_{\mathfrak{m}}^{d-1}(\mathrm{k}[\Delta])$ implies that

$$
\begin{aligned}
& H_{\mathfrak{m}}^{d-2}(\mathrm{k}[\Delta]\{1\})_{1} \cong H_{\emptyset}^{d-2}(\Delta), \\
& H_{\mathfrak{m}}^{d-2}(\mathrm{k}[\Delta]\{1\})_{0} \cong \mathscr{K}_{d-2}^{\theta_{1}}(\Delta), \\
& H_{\mathfrak{m}}^{d-2}(\mathrm{k}[\Delta]\{1\})_{i} \cong 0, \quad i \neq 0,1
\end{aligned}
$$

We can repeat this process until we produce $\theta_{1}, \ldots, \theta_{d-1}$ such that multiplication $\theta_{i}: \mathrm{k}[\Delta]\{i-1\} \rightarrow \mathrm{k}[\Delta]\{i-1\}$ is an injection for each $1 \leq i \leq d-1$ and

$$
\begin{aligned}
H_{\mathfrak{m}}^{0}(\mathrm{k}[\Delta]\{d-1\})_{d-1} & \cong H_{\emptyset}^{d-2}(\Delta), \\
H_{\mathfrak{m}}^{0}(\mathrm{k}[\Delta]\{d-1\})_{d-2} & \cong \mathscr{K}_{d-2}^{\theta_{1}}(\Delta) \cap \cdots \cap \mathscr{K}_{d-2}^{\theta_{d-1}}(\Delta), \\
H_{\mathfrak{m}}^{0}(\mathrm{k}[\Delta]\{d-1\})_{i} & \cong 0, \quad i \neq d-1, d-2 .
\end{aligned}
$$

One last prime avoidance argument to find $\theta_{d}$ such that the kernel of multiplication by $\theta_{d}$ on $\mathrm{k}[\Delta]\{d-1\}_{d-2}$ is isomorphic to $\mathscr{K}_{d-2}^{\theta_{1}}(\Delta) \cap \ldots \cap \mathscr{K}_{d-2}^{\theta_{d}}(\Delta)$ finishes the proof.

Any pure two-dimensional simplicial complex has isolated singularities. Indeed, the link of any edge is a nonempty collection of points and hence Cohen-Macaulay. As $h_{3}^{\prime}=\beta_{2}, h_{1}^{\prime}=h_{1}$, and $h_{0}^{\prime}=h_{0}=1$, it only remains to compute $h_{2}^{\prime}$.

In [16] Lee established that $h_{2}^{\prime}$ is the dimension of the space of stresses of a generic embedding of the one-skeleton of $\Delta$ into the plane. (Various definitions pertaining to rigidity theory such as those of stresses, infinitesimal motions, etc. can be found in [10].) As an algorithm for the latter for any graph, in particular for the one-skeleton of a simplicial complex, had already been determined, see [10, Theorem 4.4.3] (it is a consequence of Laman's theorem), a combinatorial formula for $h_{2}^{\prime}$ already exists. The following corollary states $h_{2}^{\prime}$ using the topology and $f$-vector $\Delta$.

COROLlary 5.2. Let $\Delta$ be a connected two-dimensional pure complex. Then for sufficiently generic $\Theta$,

$$
h_{2}^{\prime}=h_{2}+\operatorname{dim}_{k}\left(\mathscr{K}_{1}^{\theta_{1}}(\Delta) \cap \mathscr{K}_{1}^{\theta_{2}}(\Delta) \cap \mathscr{K}_{1}^{\theta_{3}}(\Delta)\right) .
$$

Proof. The 1-skeleton of $\Delta$ is CM if and only if $\Delta$ is connected.

What about two-dimensional complexes that are not connected? One can either appeal to the rigidity formula and the fact that each extra component 
introduces three new infinitesimal motions or a local cohomology argument similar to the one in Theorem 5.1 to find that the formula is

$$
h_{2}^{\prime}=h_{2}+3 \beta_{0}(\Delta)+\operatorname{dim}_{\mathrm{k}}\left(\mathscr{K}_{1}^{\theta_{1}}(\Delta) \cap \mathscr{K}_{1}^{\theta_{2}}(\Delta) \cap \mathscr{K}_{1}^{\theta_{3}}(\Delta)\right) .
$$

\section{Examples}

In this section we use methods and results developed in the paper so far to provide a complete characterization of the $f$-vectors of arbitrary simplicial triangulations of certain 3-dimensional pseudomanifolds denoted by $\left\|N_{1}\right\|$, $\left\|N_{3}\right\|$, and $\left\|N_{4}\right\|$ in [5, Example 4]. We start by recalling from [5] the necessary information on the topology of $X_{i}:=\left\|N_{i}\right\|, i=1,3,4$.

The pseudomanifold $X_{1}$ is the only normal 3-pseudomanifold with 8 singularities all of whose links are the torus that has an 8-vertex triangulation; its homology groups (over Z) are $\tilde{H}_{0}=\tilde{H}_{1}=0, \tilde{H}_{2}=\mathrm{Z}^{8}$, and $\tilde{H}_{3}=\mathrm{Z}$. Therefore, for any triangulation $\Delta$ of $X_{1}$,

$$
\begin{gathered}
h_{4}(\Delta)=-\tilde{\chi}(\Delta)=-7, \\
\sum_{v}(1-\tilde{\chi}(\operatorname{lk} v))=16, \\
h_{3}(\Delta)-h_{1}(\Delta)=16,
\end{gathered}
$$

and so

where the last equality is by Theorem 3.1.

The pseudomanifold $X_{3}$ has 5 singularities; the links of the 5 singular vertices in $N_{3}$ have different topological types: four of them are projective planes, while the fifth one is a 2-dimensional torus. The homology groups of $X_{3}$ are $\tilde{H}_{0}=\tilde{H}_{1}=\tilde{H}_{3}=0$ and $\tilde{H}_{2}=\mathrm{Z}^{2} \oplus \mathrm{Z} / 2 \mathrm{Z}$. Thus for any triangulation $\Delta$ of $X_{3}$,

$$
\begin{gathered}
h_{4}(\Delta)=-\tilde{\chi}\left(X_{3}\right)=-2, \\
\sum_{v}(1-\tilde{\chi}(\mathrm{lk} v))=6, \\
\text { hence } \quad h_{3}(\Delta)-h_{1}(\Delta)=6 .
\end{gathered}
$$

The pseudomanifold $X_{4}$ is $H \cup C(\partial H)$, where $H$ is a solid 3-dimensional torus and $C(\partial H)$ is the cone over the boundary of $H$. Hence $X_{4}$ has only one isolated singularity, and for any triangulation $\Delta$ of $X_{4}, \mathscr{K}_{2}(\Delta)=1$. In addition,

$$
\begin{aligned}
& h_{4}(\Delta)=-\tilde{\chi}(\Delta)=0, \\
& \sum_{v}(1-\tilde{\chi}(\operatorname{lk} v))=2,
\end{aligned}
$$

and so

$$
h_{3}(\Delta)-h_{1}(\Delta)=2 \text {. }
$$


As the above discussion shows, to characterize the $h$-vectors (equivalently, the $f$-vectors $)$ of arbitrary simplicial triangulations of $X_{i}(i=1,3,4)$ it is enough to characterize all possible pairs $\left(h_{1}(\Delta), h_{2}(\Delta)\right)$, where $\|\Delta\|=X_{i}$. This, in turn, is equivalent to characterizing the set

$\left\{\left(g_{1}(\Delta), g_{2}(\Delta)\right):\|\Delta\|=X_{i}\right\}, \quad$ where $g_{j}(\Delta):=h_{j}(\Delta)-h_{j-1}(\Delta)$.

To this end, we have the following result.

THEOREM 6.1. For $i=1,3,4$ and for a pair $\left(g_{1}, g_{2}\right) \in Z^{2}$, the following are equivalent:

(i) There exists a simplicial complex $\Delta,\|\Delta\|=X_{i}$ such that $\left(g_{1}(\Delta)\right.$, $\left.g_{2}(\Delta)\right)=\left(g_{1}, g_{2}\right)$

(ii) $6 \leq g_{2} \leq\left(\begin{array}{c}g_{1}+1 \\ 2\end{array}\right)$. (In particular, $g_{1} \geq 3$.)

Proof. To verify that (ii) implies (i), start with the triangulation $N_{i}$ of $X_{i}$ described in [5] (it satisfies $g_{1}\left(N_{i}\right)=3$ and $g_{2}\left(N_{i}\right)=6$ ) and follow the proof of Lemma 7.3 in Walkup's paper [34]. The triangulations $N_{i}$ (taken from [5]) and the "simple 3-trees" required to invoke this argument are listed at the top of the next page.

To prove that (i) implies (ii), fix a field $\mathrm{k}$ of characteristic zero. Consider a triangulation $\Delta$ of $X_{i}$, its face ring $\mathrm{k}[\Delta]$, a generic 1.s.o.p. for $\mathrm{k}[\Delta], \Theta=$ $\left\{\theta_{1}, \ldots, \theta_{4}\right\}$, and one additional generic linear form $\omega$. Since $\Delta$ is a normal 3dimensional pseudomanifold, it follows from [7] that $\Delta$ is generically 4-rigid in the graph-theoretic sense (see [10] or [12] for appropriate definitions). This result combined with [16, Theorem 10] yields that $\operatorname{dim}_{\mathrm{k}} \mathrm{k}(\Delta, \Theta)_{1}=h_{1}(\Delta)$, $\operatorname{dim}_{\mathrm{k}} \mathrm{k}(\Delta, \Theta)_{2}=h_{2}(\Delta)$ and the map $\cdot \omega: \mathrm{k}(\Delta, \Theta)_{1} \rightarrow \mathrm{k}(\Delta, \Theta)_{2}$ is injective. Thus for $j \leq 2, \operatorname{dim}_{\mathrm{k}}[\mathrm{k}(\Delta, \Theta) / \omega]_{j}=g_{j}(\Delta)$, and the inequality $g_{2}(\Delta) \leq$ $\left(\begin{array}{c}g_{1}(\Delta)+1 \\ 2\end{array}\right)$ follows from Macaulay's theorem (see [30, pp. 56-57]).

It only remains to show that $g_{2}(\Delta) \geq 6$. If $\Delta$ is a triangulation of $X_{4}$, then $\Delta$ has one singular vertex, and the result follows from Theorem 4.9 according to which $g_{2}(\Delta) \geq\left(\begin{array}{l}5 \\ 2\end{array}\right)[1-1+1]+4 \cdot 1-4[2-1+1]=6$.

If $\Delta$ is a triangulation of $X_{3}$, then as a k-space (rather than k-pseudomanifold) with isolated singularities, $X_{3}$ has only one singular point. (This is because over a field of characteristic 0 , the projective plane is acyclic, and hence CM.) Theorem 4.2 then implies that $\operatorname{dim}_{\mathrm{k}}(\Delta, \Theta)_{3}=h_{3}(\Delta)$. Considered as a k-pseudomanifold, $\Delta$ has only 5 singular points, and we infer from Lemma 6.2 below that the map $\omega: \mathrm{k}(\Delta, \Theta)_{2} \rightarrow \mathrm{k}(\Delta, \Theta)_{3}$ is onto, and hence $h_{2}(\Delta) \geq h_{3}(\Delta)$. Thus

$$
g_{2}(\Delta)=h_{2}(\Delta)-h_{1}(\Delta) \geq h_{3}(\Delta)-h_{1}(\Delta)=6 \quad \text { by Eq. (14). }
$$




\begin{tabular}{ccc}
\hline Triangulation & \multicolumn{1}{c}{ Facets } & Simple 3-tree \\
\hline \multirow{2}{*}{$N_{1} \quad 1248,1268,1348,1378,1568,1578}$, & $1248,1268,1348,1378,1568$ \\
& $2358,2378,2458,2678,3468,3568$, & \\
& $4578,4678,1247,1257,1367,1467$, & \\
& $2347,2567,3457,3567,1236,2346$, & \\
& $1345,1235,1456,2456$ & \\
$N_{3} \quad$ & $1248,1268,1348,1378,1568,1578$, & $1248,1268,1348,1378,1568$ \\
& $2358,2378,2458,2678,3468,3568$, & \\
& $4578,4678,1234,2347,2456,2467$, & \\
& $3456,3457,1235,1256,1357$ \\
$N_{4}$ & $1248,1268,1348,1378,1568,1578$, & $1248,1268,1348,1378,1568$ \\
& $2358,2378,2458,2678,3468,3568$, & \\
& $4578,4678,1245,1256,2356,2367$, & \\
& $3467,1347,1457$ \\
\hline
\end{tabular}

Finally, let $\Delta$ be a triangulation of $X_{1}$. Since the given triangulation $N_{1}$ of $X_{1}$ in [5] contains the complete 2-skeleton on eight vertices its depth is 3. By Theorem 5.1, $\operatorname{dim}_{\mathrm{k}} \mathrm{k}(\Delta, \Theta)_{3}=h_{3}(\Delta)$ plus a term which only depends on the topology of $X_{1}$. However, as all of the missing faces of $N_{1}$ are three-dimensional, $\operatorname{dim}_{\mathrm{k}} \mathrm{k}\left(N_{1}, \Theta\right)_{3}=h_{3}$ for this triangulation, and thus for all others. This implies that $\operatorname{dim}_{k}[k(\Delta, \Theta) / \omega]_{3} \geq g_{3}(\Delta)$, and hence that $\left(1, g_{1}(\Delta), g_{2}(\Delta), \max \left\{0, g_{3}(\Delta)\right\}\right)$ is an M-sequence. Thus according to Macaulay's theorem, if $g_{2}(\Delta)<6=\left(\begin{array}{l}4 \\ 2\end{array}\right)$, then $g_{3}(\Delta)<\left(\begin{array}{l}5 \\ 3\end{array}\right)=10$, and hence $h_{3}(\Delta)-h_{1}(\Delta)=g_{2}(\Delta)+g_{3}(\Delta)<6+10=16$, contradicting Eq. (13). Therefore, $g_{2}(\Delta) \geq 6$ in this case as well. The theorem follows.

Lemma 6.2. Let $\Delta$ be a 3-dimensional pseudomanifold with isolated singularities, $\Theta=\left\{\theta_{1}, \ldots, \theta_{4}\right\}$ a generic l.s.o.p. of $\mathrm{k}[\Delta]$, and $\omega \in \mathrm{k}[\Delta]_{1}$ a generic 1-form. If $\Delta$ has at most 5 singularities then the map $\cdot \omega: \mathrm{k}(\Delta, \Theta)_{2} \rightarrow$ $\mathrm{k}(\Delta, \Theta)_{3}$ is surjective.

Proof. Let $U \subseteq V$ be the subset of singular vertices of $\Delta$ and let $\Delta_{U}$ be the induced subcomplex. Consider the following commutative diagram.

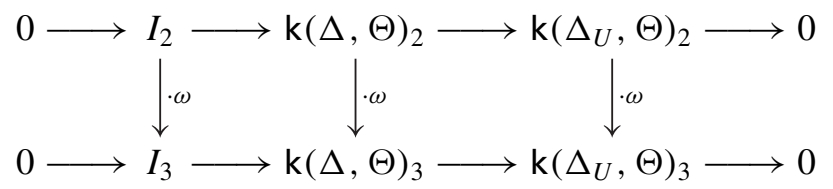

By the snake lemma it is sufficient to show that the two outside maps are surjective for generic choices of $\Theta$ and $\omega$. As $|U| \leq 5, \mathrm{k}\left(\Delta_{U}, \Theta\right)_{3}=0$ unless 
$|U|=5$ and $\Delta_{U}$ contains the entire 2-skeleton of the 4-simplex with vertex set $U$. However, if this is the case, then the right-hand map is the same as for the boundary of the 4-simplex, where it is known to be surjective. For the left-hand map, first we observe that $I_{3}$ is generated by all monomials that contain at least one nonsingular vertex. Now we follow the proof of [33, Theorem 4.26] to see that each of these monomials is in the image.

\section{PL homeomorphic pseudomanifolds}

As Theorem 4.7 shows, for a simplicial complex $\Delta$ with homologically isolated singularities, the quantities $\operatorname{dim}_{\mathrm{k}} \mathrm{k}(\Delta, \Theta)_{i}-h_{i}(\Delta)$, for $0 \leq i \leq \operatorname{dim} \Delta+1$, are independent of the choice of an 1.s.o.p. $\Theta$; moreover, they depend only on the homeomorphism type of $\|\Delta\|$. While for an arbitrary complex $\Delta$, the Hilbert function of $k(\Delta, \Theta)$ does depend on $\Theta$, for sufficiently generic linear systems of parameters it is a constant, and we define

$$
h_{i}^{\prime}(\Delta):=\operatorname{dim}_{\mathrm{k}} \mathrm{k}(\Delta, \Theta)_{i}, \quad \text { where } \Theta \text { is a generic 1.s.o.p. for } \mathrm{k}[\Delta] .
$$

Since in this section we work only with generic linear systems of parameters, to simplify the notation we write $\mathrm{k}(\Delta)$ instead of $\mathrm{k}(\Delta, \Theta)$.

A natural question raised in [18] is whether for an arbitrary $(d-1)$ dimensional complex $\Delta, h_{i}^{\prime}(\Delta)-h_{i}(\Delta)$ is determined by the homeomorphism type of $\|\Delta\|$. As noted earlier, for $i=d$, the answer is yes as $h_{d}^{\prime}(\Delta)=\beta_{d-1}(\Delta)$, [2, Lemma 2.2(3)]. The main result of this section is that for spaces with isolated singularities, $h_{i}^{\prime}-h_{i}$ is determined by the PL-homeomorphism type for all $i$. Two simplicial complexes are called PL-homeomorphic if they have a common subdivision. For other basic facts and terminology related to PL-topology we refer our readers to [24].

THEOREM 7.1. If two simplicial complexes with isolated singularities, $\Delta$ and $\Gamma$, are PL-homeomorphic, then $h_{i}^{\prime}(\Gamma)-h_{i}(\Gamma)=h_{i}^{\prime}(\Delta)-h_{i}(\Delta)$ for all $i$.

We recall that the stellar subdivision of a simplicial complex $\Delta$ at a face $F$ (where $F \in \Delta$ and $\operatorname{dim} F>0$ ) is the simplicial complex obtained from $\Delta$ by removing all faces containing $F$ and adding a new vertex $\rho$ as well as all sets of the form $\tau \cup\{\rho\}$ where $\tau$ does not contain $F$ but $\tau \cup F \in \Delta$. By Alexander's theorem [1], two simplicial complexes possess a common subdivision if and only if they possess a common subdivision obtained via successive stellar subdivisions and their inverses. Since PL-homeomorphic complexes have a common subdivision, and since the link of any face $F, \operatorname{dim} F>0$, of a complex with isolated singularities is $\mathrm{CM}$, Theorem 7.1 is a corollary to the following result. 
THEOREM 7.2. Let $\Delta$ be a $(d-1)$-dimensional simplicial complex, $F$ a face of $\Delta$ of positive dimension, and $\Gamma$ the stellar subdivision of $\Delta$ at $F$. If the link of $F$ in $\Delta, 1 \mathrm{k} F$, is Cohen-Macaulay of dimension $d-1-|F|$, then $h_{i}^{\prime}(\Gamma)-h_{i}(\Gamma)=h_{i}^{\prime}(\Delta)-h_{i}(\Delta)$ for all $i$.

Proof. Our proof is motivated by the ideas from [15]. Let $V$ be the vertex set of $\Gamma$ and $V-\{\rho\}$ the vertex set of $\Delta$. We write $\bar{F}$ to denote the simplex $F$ with all its faces. Consider the following subcomplexes:

$$
\begin{aligned}
L= & \bar{F} * \mathrm{lk} F-\text { the closed star of } F \text { in } \Delta, \\
R= & \{G \in \Delta: G \nsupseteq F\}-\text { the costar of } F \text { in } \Delta, \\
L^{\prime}= & \bar{\rho} * \partial \bar{F} * \operatorname{lk} F-\text { the join of the boundary complex of } F \\
& \text { with } \rho \text { and the link of } F .
\end{aligned}
$$

Then $\Delta=L \cup R, \Gamma=L^{\prime} \cup R$, and $L \cap R=\partial \bar{F} * \operatorname{lk} F=L^{\prime} \cap R$ - denote this common intersection by $T$. Therefore, we have the following short exact sequences of $S$-modules (recall that $S=\mathrm{k}\left[x_{v}: v \in V\right]$ ), where the maps $\phi, \psi, \phi^{\prime}, \psi^{\prime}$ are induced by inclusions of the corresponding subcomplexes:

$$
\begin{aligned}
& 0 \longrightarrow \mathrm{k}[\Delta] \stackrel{\psi}{\longrightarrow} \mathrm{k}[L] \oplus \mathrm{k}[R] \stackrel{\phi}{\longrightarrow} \mathrm{k}[T] \longrightarrow 0 \\
& 0 \longrightarrow \mathrm{k}[\Gamma] \stackrel{\psi^{\prime}}{\longrightarrow} \mathrm{k}\left[L^{\prime}\right] \oplus \mathrm{k}[R] \stackrel{\phi^{\prime}}{\longrightarrow} \mathrm{k}[T] \longrightarrow 0
\end{aligned}
$$

Let $\Theta=\left(\theta_{1}, \ldots, \theta_{d}\right)$ be an ideal of $S$ generated by $d$ generic linear forms. In particular, we can assume that $\left\{\theta_{1}, \ldots, \theta_{d}\right\}$ is an 1.s.o.p. for each of the modules $\mathrm{k}[\Delta], \mathrm{k}[\Gamma], \mathrm{k}[L]$, and $\mathrm{k}\left[L^{\prime}\right]$. (Since $\operatorname{dim} \Delta=\operatorname{dim} \Gamma=\operatorname{dim} L=$ $\operatorname{dim} L^{\prime}=d-1$, all four of these modules have Krull dimension $d$. Note however that $T$ and $R$ may have a strictly smaller dimension.)

We now tensor the above two sequences with $S / \Theta($ over $S)$. As lk $F$ is CM of dimension $d-1-|F|$, it follows that $L=\bar{F} * \mathrm{lk} F$ and $L^{\prime}=\bar{\rho} * \partial \bar{F} * \mathrm{lk} F$ are both $\mathrm{CM}$ of dimension $d-1$, and so $\mathrm{k}[L]$ and $\mathrm{k}\left[L^{\prime}\right]$ are free $\mathrm{k}\left[\theta_{1}, \ldots, \theta_{d}\right]-$ modules. The fact that for any $S$-module $M, \operatorname{Tor}_{1}^{S}(M, S / \Theta)=\operatorname{ker}\left(M \otimes_{S}\right.$ $\Theta S \rightarrow M)$, then implies that

$$
\operatorname{Tor}_{1}^{S}(\mathrm{k}[L], S / \Theta)=\operatorname{Tor}_{1}^{S}\left(\mathrm{k}\left[L^{\prime}\right], S / \Theta\right)=0 .
$$

Thus by naturality of the long exact Tor sequence, we obtain the following commutative diagram:

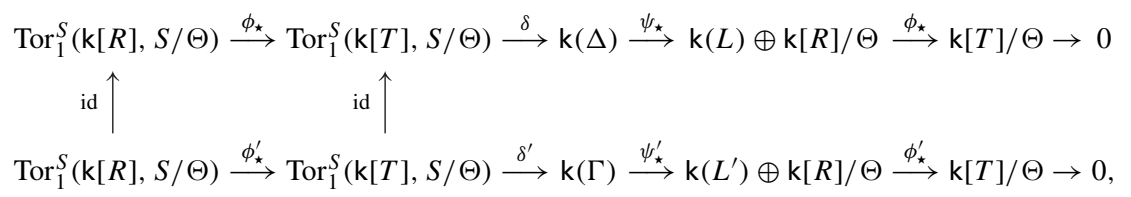


where id is the identity map, and $\delta$ and $\delta^{\prime}$ are connecting homomorphisms. Hence for all $i$,

$$
\begin{aligned}
\operatorname{dim}_{\mathrm{k}} \mathrm{k}(\Gamma)_{i}-\left(\operatorname{dim}_{\mathrm{k}}(\mathrm{k}[R] / \Theta)_{i}+\operatorname{dim}_{\mathrm{k}} \mathrm{k}\left(L^{\prime}\right)_{i}\right) \\
=\operatorname{dim}_{\mathrm{k}} \mathrm{k}(\Delta)_{i}-\left(\operatorname{dim}_{\mathrm{k}}(\mathrm{k}[R] / \Theta)_{i}+\operatorname{dim}_{\mathrm{k}} \mathrm{k}(L)_{i}\right),
\end{aligned}
$$

yielding that

$h_{i}^{\prime}(\Gamma)-h_{i}^{\prime}(\Delta)=h_{i}^{\prime}\left(L^{\prime}\right)-h_{i}^{\prime}(L)=h_{i}\left(L^{\prime}\right)-h_{i}(L)=h_{i}(\Gamma)-h_{i}(\Delta), \quad \forall i$,

as required. The second step in this computation holds since $L$ and $L^{\prime}$ are CM, and thus $h^{\prime}(L)=h(L)$ and $h^{\prime}\left(L^{\prime}\right)=h\left(L^{\prime}\right)$; the last step follows from the fact that $f_{j}(\Delta)=f_{j}(L)+f_{j}(R)-f_{j}(T)$ and $f_{j}(\Gamma)=f_{j}\left(L^{\prime}\right)+f_{j}(R)-f_{j}(T)$ for all $j$, so that $f_{j}\left(L^{\prime}\right)-f_{j}(L)=f_{j}(\Gamma)-f_{j}(\Delta)$ for all $j$, and hence also $h_{i}\left(L^{\prime}\right)-h_{i}(L)=h_{i}(\Gamma)-h_{i}(\Delta)$ for all $i$.

ACKNOWLEDGMENTS. We are grateful to Gil Kalai for numerous comments on the preliminary version of this paper.

\section{REFERENCES}

1. Alexander, J. W., Combinatorial analysis situs, Trans. Amer. Math. Soc. 28 (1926), 301-329.

2. Babson, E., and Novik, I., Face numbers and nongeneric initial ideals, Electron. J. Combin. 11:2 (2004/06), Research Paper \#25, 23 pp.

3. Barnette, D., A proof of the lower bound conjecture for convex polytopes, Pacific J. Math. 46 (1973), 349-354.

4. Chen, B., and Yan, M., Eulerian stratification of polyhedra, Adv. in Appl. Math. 21 (1998), 22-57.

5. Datta, B., and Nilakantan, N., Three-dimensional pseudomanifolds on eight vertices, Int. J. Math. Math. Sci., 2008, Art. ID 254637, 21 pp.

6. Eisenbud, D., Commutative Algebra. With a View Toward Algebraic Geometry, Grad. Texts in Math. 150, Springer, New York 1995.

7. Fogelsanger, A., The generic rigidity of minimal cycles, $\mathrm{PhD}$. thesis, Cornell Univ. 1988.

8. Goresky, M., and MacPherson, R., Intersection homology theory, Topology 19 (1980), 135162.

9. Gräbe, H.-G., The canonical module of a Stanley-Reisner ring, J. Algebra 86 (1984), 272-281.

10. Graver, J., Servatius, B., and Servatius, H., Combinatorial Rigidity, Grad. Studies in Math. 2, Amer. Math. Soc., Providence, RI 1993.

11. Hersh, P., and Novik, I., A short simplicial h-vector and the upper bound theorem, Discrete Comput. Geom. 28 (2002), 283-289.

12. Kalai, G., Rigidity and the lower bound theorem I, Invent. Math. 88 (1987), 125-151.

13. Klee, V., A combinatorial analogue of Poincaré's duality theorem, Canad. J. Math. 16 (1964), 517-531.

14. Klee, V., On the number of vertices of a convex polytope, Canadian J. Math. 16 (1964), 702-720.

15. Kubitzke, M., and Nevo, E., The Lefschetz property for barycentric subdivisions of shellable complexes, Trans. Amer. Math. Soc. 361 (2009), 6151-6163. 
16. Lee, C. W., Generalized stress and motions, pp. 249-271 in: Polytopes: Abstract, Convex and Computational, Proc. Scarborough, ON 1993, NATO Adv. Sci. Inst. C 440, Kluwer, Dordrecht 1994.

17. McMullen, P., The maximum numbers offaces of a convex polytope, Mathematika 17 (1970), 179-184.

18. Miller, E., Novik, I., and Swartz, E., Face rings of simplicial complexes with singularities, Math. Ann. 351 (2011), 857-875.

19. Murai, S., Algebraic shifting of strongly edge decomposable spheres, J. Combin. Theory A 117 (2010), 1-16.

20. Novik, I., Upper bound theorems for homology manifolds, Israel J. Math. 108 (1998), 45-82.

21. Novik, I., and Swartz, E., Applications of Klee's Dehn-Sommerville relations, Discrete Comput. Geom. 42 (2009), 261-272.

22. Novik, I., and Swartz, E., Socles of Buchsbaum modules, complexes and posets, Adv. in Math. 222 (2009), 2059-2084.

23. Reisner, G., Cohen-Macaulay quotients of polynomial rings, Adv. in Math. 21 (1976), 30-49.

24. Rourke, C. P., and Sanderson, B. J., Introduction to Piecewise-Linear Topology, Ergebn. Math. Grenzgeb. 69, Springer, Heidelberg 1972.

25. Schenzel, P., On the number of faces of simplicial complexes and the purity of Frobenius, Math. Z. 178 (1981), 125-142.

26. Smith, D. E., On the Cohen-Macaulay property in commutative algebra and simplicial topology, Pacific J. Math. 141 (1990), 165-196.

27. Stanley, R., The upper bound conjecture and Cohen-Macaulay rings, Studies in Applied Math. 54 (1975), 135-142.

28. Stanley, R., Cohen-Macaulay complexes, pp. 51-62 in: M. Aigner (ed.), Higher Combinatorics, NATO Adv. Study Inst. C 31, Reidel, Dordrecht 1977.

29. Stanley, R., The number of faces of a simplicial convex polytope, Adv. in Math. 35 (1980), 236-238.

30. Stanley, R., Combinatorics and Commutative Algebra, 2nd ed., Progress in Math. 41, Birkhäuser, Boston 1996.

31. Stückrad, J., and Vogel, W., Buchsbaum Rings and Applications, Springer, Berlin, 1986.

32. Swartz, E., Lower bounds for h-vectors of k-CM, independence, and broken circuit complexes, SIAM J. Discrete Math. 18 (2004/05), 647-661.

33. Swartz, E., Face enumeration - from spheres to manifolds, J. Europ. Math. Soc. 11 (2009), 449-485.

34. Walkup, D. W., The lower bound conjecture for 3-and 4-manifolds, Acta Math. 125 (1970), 75-107.

35. Wall, C. T. C., Arithmetic invariants of subdivision of complexes, Canad. J. Math. 18 (1966), 92-96.

36. Whiteley, W., Vertex splitting in isostatic frameworks, Structural Topology 16 (1990), 23-30.

DEPARTMENT OF MATHEMATICS

BOX 354350

UNIVERSITY OF WASHINGTON

SEATTLE, WA 98195-4350

USA

E-mail: novik@math.washington.edu
DEPARTMENT OF MATHEMATICS

CORNELL UNIVERSITY

ITHACA, NY 14853-4201

USA

E-mail: ebs22@cornell.edu 